Zeitschrift für Augenheilkunde 1919;42:I-IV

\title{
Contents, Vol. 42, 1919
}

\section{Inhalts-Verzeichnis.}

Seite

Origînal-Arbeiten.

Asmus, Die Zinkiontophorese bei Ulcus serpens 216

Bergmeister, R., Über Augenmuskellähmungen in der frühen

Sekundärperiode der Syphilis 22

Cramer, E., Wiederherstellung der zerstörten Bindehautsäcke

beider Augen dureh Stentsplastik 116

Eyer, A.. Zwei Fälle von Herpes zoster ophthalmicus . . . 234

Farid, Nasr., Beitrag zum Verhalten des Visu1/1/8 bei Astig-

matismus õõ

Fuchs, A., Über die Entstehung einer reflektorischen Pupillen-

starre durch Methylalkoholvergiftung

50

Qaüus, E., Über Hornhautastigmatisnms, insbesondere überdas Vorkornmen und die

pathologische Bedeutung seinerperversen Form bei Diabetes und anderen Allgemein-

erkrankungen 203

Hen-sen, H., Über die Wirkung kutaner ïuberkulinimpfungennaeh Ponndorf auf skrophulöse und tuberkulöse Augen-erkrankungen 221

He-ÿò·e, R., Über Vorfall von Glaskörper in die Vorderkammer 191

Junius, Doppelseitige Erblindung nach Gesichtserisypel mit seltenem Befund an der Netzhaut. (Hierzu Tafel I.) . . 1

Kirsch, R., Ein typischer Fall von Bindehautschürze (Epitarsus). (Hierzu ïafel II.)

60

Kraupa, E., Über Behandlungsresultate tuberkulöser Augen-affektionen bei Anwendung von Partialantigenen undMilchinjektionen 105

Kuhnt, H., Einige Bemerkungen zur Arbeit von Herrn G. Levin-sohn „Zur Frage der Diszission des Nachstars" .... 247

Levinsohn, Zur Frage der Diszission des Nachstars .... 241

Lindner, K., Über die Topographie der Bindehautkeime . . 11

Lutz, A., Die Augensymptome bei Pseudotumor-Cerebri nebst Mitteilung einer neuen Beobachtung. (Rückbildung von bilateraler Stauungspapille zur Norm, Entwicklung von einseitigem Romberg und von einseitiger Taubheit, der ein Jahr später der Verlust der Vertibularisfunktion folgte.) 166

$-\mathrm{IV}-$

Sßite

Podesta, Die G $\pi$ mdlagen der Ostwaldschen Farbenlehre in

ophthalmologiseher Beleuchtung 273

Schiller, E., Über Sarkome der Augenlider 302

Schöppe, H., Ein Pall von Neuritis retrobulbaris während der 
Laktation

316

Simon, P., Refraktion und Rriegsbrauchbarkeit

64

Stargardt, K., Über eine postoperative Panophthalmie dureh

gramnegative Mikrokokken 227

Tertsch, R., Die Arasio conjunctivae als Heilmittel des Trachoms 34

Priv.-Doz. Dr. JW $3 / 8 \frac{1}{2}<$ s-Bonn, Gutachten über die Prage des $7 / 8$ usammenhanges von Thrombose der Zentralvene der Netzhaut eines Augt > s niit Ruhr bzw. Malaria im Kriege 122

Gesellsehaftsberichte.

Ophthalmologisehe Gesellschaft in Wien. Sitzung vom

2. Juni 1919 und 7. Juli 1919 97, 253

Literaturberichte.

Mikroorganismen und Serologie (ausschl. Diagnose undTherapie). Bericht über die Jahre $1914^{\wedge}$ 1918. VonProf. Dr. Zade in Heidelberg 83

Diagnostik und Extraktion von Fremdkörpern. Bericht überdie Jahre 1914-1918. Von San.-Rat.

Dr. Asmns inDüsseldorf 127

Glaukom, Motilitätsstörungen und sympathische Ophthalmie. Bericht über die deutsche

ophthalmologisehe Literatur des Jahres 1918. Von Prof. Dr. C. H. Saltier in Königsberg 150

Unfall- und Versieherungskunde 159, 267, 323

Diagnostische Notizen 265

Literatur-Verzeichnis 162, 325

Personalien $\quad 104$

P. Preisaufgabe 271

Teehnischer Aussehuß íür Brillenoptik (Tabo) 272 